\title{
Wirksamkeit und Sicherheit der sublingualen Immuntherapie (SLIT) - eine ausführliche Standortbestimmung
}

Efficacy and Safety of Sublingual Immunotherapy - a Detailed Analysis

\section{Zusammenfassung}

Die sublinguale Immuntherapie wurde entwickelt, um das Risiko schwerer systemischer Nebenwirkungen zu vermeiden, wie sie bei der subkutanen Immuntherapie auftreten können. Auf der Grundlage von mehr als 20 kontrollierten Studien kann festgestellt werden, dass die Wirksamkeit und Sicherheit der SLIT zur Behandlung der allergischen Rhinokonjunktivitis bei Pollenund Milbenallergie mittlerweile gut belegt ist. Die Daten für das allergische Asthma bedürfen noch der Überprüfung. Die sublinguale Immuntherapie stellt keinen Ersatz der subkutanen Immuntherapie dar, sondern ist eine relevante zusätzliche Option für definierte Patientengruppen. Von entscheidender Bedeutung für die Wirksamkeit ist die Applikation einer ausreichend hohen Dosis des Allergenextraktes. Für den klinischen Alltag kann die Empfehlung der ARIA-Working-Group zur Anwendung einer mindestens 50- bis 100-fachen kumulativen Dosis im Vergleich zur subkutanen IT verwendet werden. Die Therapie kann präoder cosaisonal sowie perennial erfolgen. Die Titration bei Behandlungsbeginn erfolgt heute üblicherweise mit täglich steigender Dosis über wenige Tage, bei ausgesuchten erwachsenen Patienten mit einer Pollenallergie gegebenenfalls auch innerhalb weniger Stunden. Die gut tolerierte Erhaltungsdosis wird im Allgemeinen während der gesamten Behandlungsdauer drei mal pro Woche oder täglich eingenommen. Nach endgültiger Validierung könnte die sublinguale saisonale Kurzzeit-Immuntherapie eine zusätzliche Option für bestimmte Patientengruppen, wie zum Beispiel Jugendliche, darstellen.

\section{Abstract}

Sublingual immunotherapy (SLIT) was developed to avoid the risk of severe systemic side effects which occur in subcutaneous immunotherapy. Data from more than 20 controlled studies clearly show the efficacy and safety of this type of immunotherapy in patients with allergic rhino-conjunctivitis due to pollen and mites. The data for allergic asthma still need confirmation. Sublingual immunotherapy is no substitute for subcutaneous immunotherapy but an additional option for defined groups of patients. The application of a sufficient amount of allergen is important for the efficacy of SLIT. According to the recommendation of the ARIA working group, a 50- to 100 -fold cumulative dose should be applied as compared to subcutaneous immunotherapy. SLIT can be used preseasonally, during the saison or perennially. Therapy starts with a daily increase of the dose. In some cases, e.g., in adult patients with pollen allergy, doses can be increased within hours. The well-tolerated maintenance dose should be taken three times a week or daily. The sublingual seasonal short-time immunotherapy may become an additional option for subgroups of patients, e.g., for adolescents. 
Einleitung

Die Zahl der Patienten mit allergischer Rhinitis oder allergischem Asthma gegen Aeroallergene nimmt kontinuierlich zu. Man geht davon aus, dass heute in der Bundesrepublik Deutschland etwa ein Drittel der Bevölkerung von einer der beiden Erkrankungen betroffen ist und dass die Zahl der Betroffenen kontinuierlich zunimmt [1]. Die subkutane Immuntherapie (SCIT) ist eine etablierte Behandlungsform für diese Erkrankungen, aber das Risiko für auch schwer verlaufende Nebenwirkungen bis hin zu solchen mit letalem Ausgang [2] limitiert den breiten Einsatz bei einer großen Zahl von Patienten. Daher rücken alternative Applikationswege zunehmend in den Blickpunkt des akademischen Interesses, wobei die sublinguale Immuntherapie (SLIT) heute am besten untersucht ist. Es liegen bereits zahlreiche Reviews und Meta-Analysen internationaler Experten und Arbeitsgruppen vor [3-8]. Auch in Deutschland wurde die wissenschaftliche Diskussion gestartet [9].

Eine einstimmige Meinung der Expertengruppen zur SLIT steht noch aus. Die Gründe hierfür sind vielfältig. So unterscheidet sich die Auswahl der betrachteten Studien in den verschiedenen Auswertungen. Für die Evaluation wurden verschiedenste Methoden von einer arbiträren Auswertungsstrategie [7,8] bis zu evidenzbasierten systematischen Verfahren [5] verwendet. Beides stellt möglicherweise einen „Bias“ dar. Ein weiterer wichtiger Punkt scheint aber die verwendete Dosis zu sein. Die Wichtigkeit einer ausreichend hohen Dosis für eine klinisch relevante Wirksamkeit wurde bereits in einem internationalen Review dargelegt [4] und am Modell des Wiesenlieschgrases weiter verdichtet [10]. Daher soll im Folgenden das Datenmaterial zur SLIT für die allergische Rhinitis und das allergische Asthma unter diesem Blickwinkel immunologisch und klinisch betrachtet werden.

\section{Immunologische Mechanismen}

Pollen und Sporen sowie Makromoleküle und Proteine in biologisch und immunologisch aktiver Form werden unter physiologischen Bedingungen durch die intakte Schleimhaut hindurch resorbiert [11 - 15], wobei die Aufnahme von Makromolekülen allergologisch relevanter Größe bei atopischen Patienten höher ist als bei Gesunden [16-18]. Somit können Allergene in immunologisch wirksamer Form und in relevanter Menge durch die Schleimhaut hindurch resorbiert werden. Die Phänomene der immunologischen Auseinandersetzung des Organismus mit den sublingual applizierten Allergenen sind noch nicht vollständig aufgeklärt. Es existieren aber bereits einige Modelle zum Wirkmechanismus. Aus zahlreichen Studien lässt sich ableiten, dass sich die immunologischen Effekte in Abhängigkeit von der applizierten Dosis deutlich unterscheiden. So führt die Gabe hoher Antigendosen (Ovalbumin) in sensibilisierten Ratten zu einer Verringerung der IgE-Produktion [19]. Die Gabe niedriger Allergendosen führt dahingegen zu einer Erhöhung [20]. Eine Studie an Patienten konnte zeigen, dass die hochdosierte sublinguale Allergenapplikation den saisonalen IgE-Anstieg in der nasalen Mukosa vermeiden kann, nicht aber die Gabe einer niedrigen Dosis [21]. Eine große Menge an Allergen steigert die Allergenaufnahme durch die dendritischen Zellen in der oralen Mukosa und somit den Grad der T-Lymphozyten-Stimulation. Auch die T-ZellAntwort wird durch die Antigenmenge beeinflusst [22]. So lösen niedrige Dosierungen eher eine Th2-Antwort mit der Produktion von IL-4 und IL-13 und somit einer bevorzugten IgE-Produktion durch B-Lymphozyten aus, während große Antigenmengen eher eine Th1-Antwort mit der Produktion von IFN- $\gamma$ auslösen und wahrscheinlich regulatorische CD4- und CD25-positive T-Zellen stimulieren, so dass die Produktion von TGF- $\beta$ und IL-10 gesteigert wird $[20,23]$.

In klinischen Studien ergibt sich aber noch kein klares Bild hinsichtlich immunologischer Parameter. Während das spezifische IgE in einigen Studien durch die sublinguale IT nicht beeinflusst zu werden scheint [24-26], zeigt sich in anderen Studien ein signifikanter Anstieg sowohl des spezifischen IgE's alleine [27] als auch zusammen mit IgG4 [28]. Ein signifikanter Anstieg in den IgG-Subklassen wurde für IgG1 [24], IgG4 [24,25,29,30] und für das Verhältnis IgG4/IgG1 [24] beobachtet, während sich in einer anderen Studie keine Veränderung der IgG4-Antikörper zeigte [26]. Darüber hinaus gibt es wie auch bei der subkutanen Hyposensibilisierung keinerlei Zusammenhang mit immunologischen Parametern und Veränderungen im klinischen Bild des Patienten.

\section{Pharmakokinetik der SLIT}

Bis vor kurzem gab es nur wenige Daten zur Pharmakokinetik lokaler Allergenextrakte. Untersuchungen an Tieren und am Menschen, im Wesentlichen mit der nasalen Applikation, weisen auf eine signifikante Resorption des Antigens über die Mukosa hin. Die Resorption bei topischer Verabreichung ist im Vergleich zur parenteralen Verabreichung geringer. Die Menge des resorbierten Antigens ist jedoch ausreichend hoch, um eine Reaktion hervorzurufen [31].

Nachdem sie zunächst beim Tier nachgewiesen wurde [32], konnte die systemische Resorption von Allergenen nach topischer Verabreichung auch beim Menschen bestätigt werden [33]. Das gereinigte Parietaria-judaica-Majorallergen Par j1 wurde radioaktiv mit ${ }^{123}$ I markiert und über den sublingualen Weg über 30 Minuten, ohne es herunter zu schlucken, an gesunde Probanden verabreicht. Die eher unrealistische Verweildauer stand in Zusammenhang mit einem der Studienziele, nämlich der Beurteilung der Allergenpenetration durch die sublinguale Schleimhaut. Das Allergen wurde nicht durch den Speichel abgebaut, und es konnte keine direkte Resorption durch die sublinguale Schleimhaut nachgewiesen werden, da die Plasmaradioaktivität erst nach dem Herunterschlucken anstieg. Darüber hinaus war eine relevante Menge ${ }^{123}$ I-markierten Par j1 noch über $48 \mathrm{~h}$ nach Verabreichung im sublingualen Bereich nachweisbar, während das freie ${ }^{123}$ I schnell eliminiert wurde. Dieser Umstand weist auf eine lokale Verarbeitung des Antigens hin und könnte für den Wirkmechanismus der SLIT von Bedeutung sein.

\section{Klinische Wirksamkeit}

Die Allergenextrakte werden als wässrige Lösungen vom Patienten zu Hause eingenommen. Die Extrakte werden in der Induktionsphase täglich und während der Erhaltungstherapie drei- bis siebenmal wöchentlich appliziert. Es liegen auch schon erste Daten für die Verkürzung der Induktionsphase auf wenige Stunden vor. Heute wird nur noch die sublingual-orale Applikation („sublingual-swallow“) empfohlen, da nur dieser Methode sowohl im WHO-Positionspapier zur spezifischen Immuntherapie [3] als auch im ARIA-Workshop-Report [4] eine Wirksamkeit zugespro- 
Tab. 1 Übersicht zu kontrollierten Studien mit der SLIT mit Angabe der kumulativen Dosis

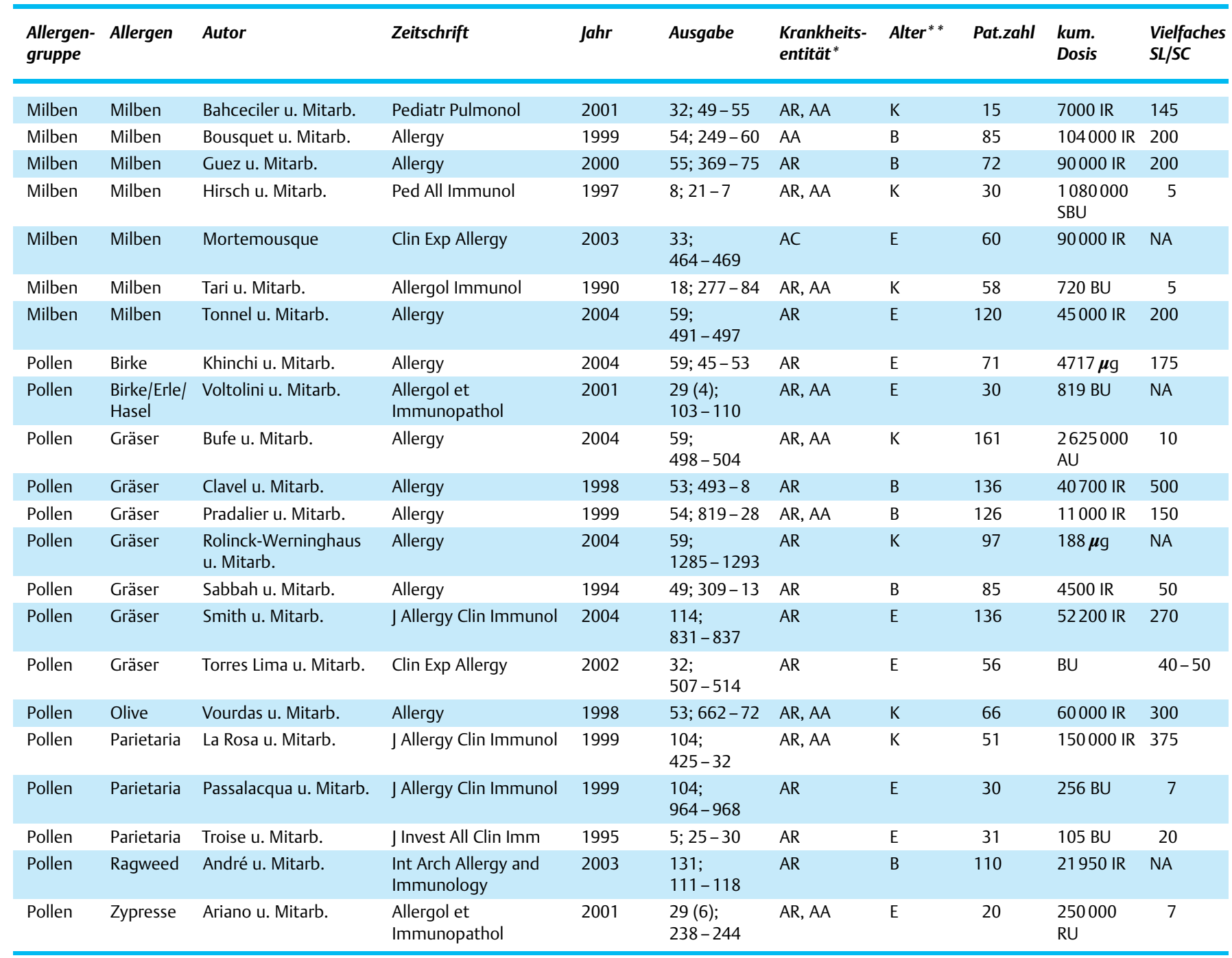

*AA = allergisches Asthma, AR = allergische Rhinitis, $A C=$ allergische Konjunktivitis

${ }^{* *} \mathrm{E}=$ Erwachsene, $\mathrm{K}=$ Kinder, $\mathrm{B}=$ beide Altersgruppen

chen wird. Hierbei wird der Extrakt zur Resorption durch die oralen Schleimhäute für ein bis zwei Minuten im Mund behalten und anschließend heruntergeschluckt.

Für die folgende Betrachtung der Wirksamkeit mussten alle berücksichtigten Studien diesen Kriterien genügen:

- Plazebo-kontrolliertes, doppelblindes Studiendesign (DBPCStudien)

- Publikation in „peer-reviewed“-Zeitschriften auf Englisch

- Definierte Allergenextrakte (Pollen oder Milben) und Dosierungen

- „Sublingual-swallow“-Applikation mit nativen Allergenen

- Klinische Wirksamkeit (Symptom-, Medikamentenscore, Provokationstests) als primäre Zielparameter

Insgesamt wurden in einer Medline-Recherche 22 Studien gefunden (s. Tab.1). Diese untersuchten die Wirksamkeit der SLIT bei der allergischen Rhinitis und/oder dem allergischen Asthma gegen Birkenpollen [34,35], Olivenpollen [26], Zypresse [36], Gräserpollen [29,37-42], Parietaria [25,30,43], Zwergambrosie [44], Hausstaubmilben $[24,27,28,45-48]$. Insgesamt wurden in diesen Studien 1646 Patienten untersucht. In sieben Studien waren ausschließlich Kinder eingeschlossen, in neun Studien ausschließlich Erwachsene und in sechs Studien Erwachsene und Kinder.

Die gewählten Zielkriterien unterscheiden sich bei den Studien teilweise erheblich hinsichtlich Auswahl und Score-Definition. Dies macht einen Vergleich schwierig. Bei einigen Publikationen wurde allerdings der aus Symptomverbesserung und Medikamentenreduktion bestehende Gesamtscore angegeben. Hier fällt auf, dass bei Dosierungen unterhalb der von der ARIA empfohlenen Minimalgabe des 50-fachen der subkutanen Dosis die beobachteten, teilweise nicht signifikanten Veränderungen zwischen 15 und $23 \%[34,39,40,43]$ niedriger ausfielen als die angegebene Verbesserung von $50 \%$ bei einer höher dosierten Sublingualtherapie [35]. So entsprach die Monatsdosis von etwa $465 \mu \mathrm{g}$ Bet v1 hier der Gesamtdosis über 5 Monate in der Studie von Voltolini u. Mitarb., worunter es zu einer nicht signifikanten Reduktion des Gesamtscores von etwa 16\% im Vergleich zu Plazebo kam [34]. 
Bei den Einzelscores sind die Veränderungen im Hinblick auf die applizierte Dosis nicht immer so konsistent. Schon in einer der ersten Studien zur sublingualen Immuntherapie an 85 Patienten mit einer Gräserpollenallergie, bei der noch mit einer recht geringen Dosierung gearbeitet wurde, zeigte sich eine Verbesserung der rhinitischen Beschwerden. Obwohl die kumulative Dosis lediglich 50-mal höher als bei s.c.-Applikation war, zeigte sich eine Reduktion der rhinitischen Beschwerden insbesondere zu den Zeiten des maximalen Pollenflugs, wobei z.B. der Score für „Niesen“ um etwa 30-50\% reduziert werden konnte. Diese Symptomverbesserung wurde von einer deutlichen Verringerung der Medikamenteneinnahme begleitet. So lag die Zufuhr oraler Kortikoide unter Verum mit um $90 \%$ unter dem Verbrauch der Plazebo-Gruppe $(\mathrm{p}<0,01)$ [37]. Der Medikamentenverbrauch war Hauptzielparameter in einer weiteren Studie an 136 Patienten mit einer allergischen Rhinokonjunktivitis durch Graspollen. Die Patienten sollten ihre Symptome durch Einnahme von AntiSympathomimetika soweit als möglich reduzieren. Dies wurde in beiden Gruppen erreicht, wie die vergleichbaren Symptomscores zeigen. Die AUC des Medikationsscores lag aber in der Behandlungsgruppe um fast $70 \%$ unter der der Kontrollgruppe $(p<0,01)$. Der Bedarf an Betamethason lag in der Behandlungsgruppe um $80 \%$ unter dem der Plazebogruppe $(p<0,01)$. In dieser Studie war die kumulative Allergendosis 500-mal höher als die subkutan gegebene [29]. In einer Kinderstudie an 97 Patienten mit Gräserpollenallergie kam es zu nicht-signifikanten ScoreVerbesserungen zwischen 0 und $31 \%$ im Vergleich zu Plazebo bei Augen-, Nasen- und Bronchialsymptomen sowie einer Reduktion des Medikamentenverbrauchs von $43 \%(p=0,0025)$. Die Dosis war in dieser Studie etwa 1,5-mal höher als bei der s.c.-Gabe [40]. Dies legt wiederum die Vermutung nahe, dass das Ausmaß der Wirksamkeit auch bei einer Gräserpollenallergie von der Dosis abhängt. Andererseits waren in 2 Tablettenstudien mit einer 150- bzw. 270-fach höheren Dosis im Vergleich zur s.c.-Gabe die Verbesserungen der Rhinitis- bzw. Medikationsscores zwischen etwa 20 und $45 \%$ ebenfalls nur moderat ausgeprägt $[38,41]$. Inwieweit dies durch die andere Galenik oder den Einschluss von therapierefraktären Patienten [41] bedingt ist, kann nicht sicher beurteilt werden.

In einer Studie an 41 Kindern mit einer allergischen Rhinokonjunktivitis infolge Parietariapollen wurde nachgewiesen, dass die Allergenschwellendosis bei der konjunktivalen Provokation positiv beeinflusst wird. Bereits nach einjähriger Therapie war die zum Auslösen allergischer Reaktionen notwendige Allergenmenge mehr als doppelt so hoch [30]. Auch bei der nasalen Provokation mit Parietaria verdoppelte sich der Schwellenwert in einer weiteren Studie [43]. Für perenniale Allergene konnte eine Verdreifachung des Schwellenwertes zur Auslösung konjunktivaler Reaktionen bei einer Milbenallergie gezeigt werden [47].

Eine interessante Therapieoption für saisonale Allergene stellt die Ultra-Rush-Titration zu Beginn des Pollenflugs dar, bei der die Erhaltungsdosis innerhalb von 2 Stunden erreicht wird. Die Wirksamkeit der saisonalen Therapie mit Titration über mehrere Tage konnte bereits vor längerem gezeigt werden [26]. Nun liegen erste Daten aus kontrollierten Studien an Erwachsenen vor, welche eine gute Verträglichkeit $[49,50]$ und Wirksamkeit $[50,51]$ dieses neuen Behandlungsschemas zeigen.
Bei der Milbenallergie sind die Ergebnisse unter sublingualer Behandlung häufig weniger eindrücklich, wobei die negativen Ergebnisse bei den Symptom- und Medikationsscores nicht selten ebenfalls durch eine niedrige Dosis bedingt zu sein scheinen $[45,46]$, aber auch durch gleichzeitige Expositionsprophylaxe verursacht sein können [27]. Bei einer ausreichend hohen Dosis kann die SLIT aber auch bei Patienten mit trotz Expositionsprophylaxe weiter bestehender ausgeprägter Symptomatik zu einer deutlichen Verbesserung des klinischen Bildes führen. So konnten die Rhinitisscores in einer zweijährigen Studie um ca. 50\% reduziert werden, wobei eine Abnahme der Symptome bereits im ersten Monat beobachtet wurde [48].

Auch wenn die Datenlage für das allergische Asthma noch nicht so eindeutig ist wie für die Rhinitis, so konnte eine Wirksamkeit der SLIT bei Asthma bereits in mehreren Studien demonstriert werden. In einer Studie an 66 Kindern mit einer Baumpollenallergie war der Dyspnoe-Score in der Verumgruppe in beiden Behandlungsjahren um ca. 50\% niedriger als in der Plazebogruppe [26]. In einer weiteren Studie zeigte sich eine Verringerung der Tage mit Asthmabeschwerden um mehr als 70\% im Vergleich zur Plazebogruppe. Nicht nur die Zahl der Asthma-Tage verringerte sich, auch die Zahl der Patienten, die überhaupt noch Asthma-Symptome hatten, war mit $15 \%$ in der Verumgruppe deutlich geringer als in der Plazebogruppe mit fast $40 \%(\mathrm{p}<0,005)$ [38]. Dies wurde bereits vorher in einer Untersuchung ebenfalls an Patienten mit einer Gräserpollenallergie gezeigt. Hier persistierte das Asthma unter der Therapie bei $10 \%$ der behandelten Patienten vs. $50 \%$ der Patienten ohne SLIT $(p<0,02)$ [29]. In einer zweijährigen Untersuchung an Patienten mit Milbenasthma zeigten sich auch Einflüsse der SLIT auf Lungenfunktionsparameter, wenngleich sich kein Korrelat bei den Symptom- und Medikationsscores fand. Es verbesserten sich der morgendliche und abendliche Peak-Flow, Vitalkapazität, $\mathrm{FEV}_{1}$ und unspezifische Hyperreagibilität im Vergleich zur Ausgangssituation in der Verumgruppe signifikant, während in der Plazebogruppe keine signifikanten Veränderungen zu beobachten waren. Dies stand in Übereinstimmung mit der beobachteten Verbesserung der Lebensqualität in den "Items“ allgemeiner Gesundheitszustand, Schmerzen und Rollenverhalten wegen körperlicher Funktionsbeeinträchtigung (alle $p=0,01$ ) in der Verumgruppe [28]. Auch für das allergische Asthma fällt auf, dass negative Ergebnisse häufig in Studien mit einer eher niedrigen Dosis beobachtet werden $[40,46]$.

Die sublinguale und die subkutane Immuntherapie wurden bereits in mehreren kontrollierten Studien verglichen, wobei methodische Mängel eine eindeutige Interpretation der Daten erschwerten [52-55]. Beide Applikationsformen wurden nun aber in einer randomisierten doppel-blinden, Plazebo-kontrollierten „double-dummy“-Studie verglichen. In der SLIT-Gruppe war die kumulative Allergendosis hierbei 200-mal höher als in der SCIT-Gruppe. Sowohl SLIT als auch SCIT zeigten eine signifikant bessere Wirkung im Vergleich zu Plazebo, wobei diese Besserung des klinischen Bildes um $50 \%$ in der SLIT-Gruppe und um $66 \%$ in der SCIT-Gruppe im direkten Vergleich nicht signifikant unterschiedlich waren. In der SCIT-Gruppe kam es allerdings zu mehreren schweren systemischen Reaktionen bis hin zur Anaphylaxie, während in der SLIT-Gruppe nur leichte bis mäßige Lokalreaktionen im Mundbereich sowie einige leichte rhinokon- 
junktivitische Reaktionen zu beobachten waren. Die Autoren kamen daher zu dem Schluss, dass methodisch bedingt zwar keine Äquivalenz von SLIT und SCIT herausgearbeitet werden konnte, dass aber aufgrund des überlegenen Sicherheitsprofils die SLIT bevorzugt werden sollte [35].

\section{Offene Fragen zur klinischen Wirksamkeit}

Wenngleich sich die Bedeutung einer ausreichend hohen Dosis zum Erzielen eines relevanten therapeutischen Effekts aus den bisherigen Studien bereits ableiten lässt, so steht doch der eindeutige Beleg noch aus. Zwar wurden 2004 auf der Jahrestagung der europäischen Akademie für Allergologie und klinische Immunologie (EAACI) erste Daten einer Dosisfindungsstudie präsentiert, die die ARIA-Empfehlungen zur Dosis zu bestätigen scheinen [56], die vollständige Publikation dieser Daten steht aber aus. Auch noch nicht vollständig geklärt sind die Fragen zur Langzeit-Wirksamkeit und zum präventiven Effekt. Es existieren aber bereits Daten aus klinischen Studien, die erste Hinweise geben. Hinsichtlich des Langzeiteffekts konnte in einer kontrollierten Studie an 60 Kindern mit allergischem Milbenasthma gezeigt werden, dass die über vier bis fünf Jahre durchgeführte Behandlung mit SLIT zu einer deutlichen und signifikanten Reduktion des Asthma-Schweregrads führte, welcher über die folgenden fünf Jahre unverändert bestehen blieb [57]. Auch für präventive Effekte gibt es erste Hinweise. So war in einer offenen Studie an Kindern mit Rhinokonjunktivitis über drei Jahre die Zahl der Patienten mit einem neu manifestierten Asthma in der Behandlungsgruppe doppelt so hoch wie in der Kontrollgruppe [58]. In einer weiteren, ebenfalls dreijährigen Untersuchung waren Neusensibilisierungen gegen weitere Aeroallergene in der Kontrollgruppe ebenfalls wesentlich häufiger als in der Behandlungsgruppe [59]. Konfirmatorische, randomisierte, doppelblinde, plazebokontrollierte Studien an einer ausreichenden Patientenzahl stehen aber noch aus.

\section{Sicherheit der SLIT}

Das gute Sicherheitsprofil zeigte sich auch in allen anderen Studien zur sublingualen Hyposensibilisierung. So wurden bisher keine schweren systemischen Nebenwirkungen beobachtet. Am häufigsten sind lokale Nebenwirkungen mit Juckreiz im HalsNasen-Mundbereich, seltener gastrointestinale Symptome wie Übelkeit, Erbrechen oder Magenschmerzen.

In einer frühen Studie mit Kindern an Rhinitis und Asthma ( $\mathrm{n}$ für Verum $=30$ ) fanden sich 32 Episoden mit systemischen Nebenwirkungen (3 Patienten mit Urtikaria, 11 Patienten mit Asthma, davon 3 schweres Asthma und 4 Patienten mit Diarrhoe) [24]. Es ergeben sich jedoch Hinweise darauf, dass die scheinbaren Unterschiede zwischen der Verum- und der Plazebo-Gruppe hauptsächlich auf zwei Ursachen zurückzuführen sind: 1. eine schlechte Dosisanpassung bei einigen Patienten, wobei es nach Dosisreduktion zu einem Verschwinden der genannten Nebenwirkungen kam und 2. eine unvollständige Darstellung der Ergebnisse, da die in der Plazebogruppe auftretenden Symptome im Gegensatz zu denen in der SLIT-Gruppe nicht einzeln aufgeführt wurden. Darüber hinaus ist es schwer zu erkennen, ob es sich bei den angegebenen Werten um die Anzahl der Patienten oder der Ereignisse handelt. Die Beobachtungen von Tari u. Mitarb. ließen sich in zahlreichen anderen Studien dann auch nicht bestätigen. So berichteten Di Rienzo u. Mitarb. [60] über ihre Er- fahrungen in der Behandlung von 268 Kindern, die eine SLITTherapie einer Dauer von 3 Monaten bis 7 Jahren (Durchschnitt: 34 Monate) erhielten, was etwa 96000 Einnahmen des Extrakts entspricht. Es wurden keine schweren systemischen Wirkungen genannt und nur 8 Nebenwirkungen verzeichnet (abdominelle Schmerzen, juckende Konjunktiven, Rhinitis, Urtikaria).

In einer unlängst publizierten Meta-Analyse zur Sicherheit der „Sublingual-swallow-“ Immuntherapie zeigten sich in den 8 untersuchten DBPC-Studien ebenfalls keine schweren unerwünschten Ereignisse oder gar anaphylaktische Reaktionen. Leichte lokale Reaktionen im Mundbereich und Gastrointestinaltrakt traten bei Erwachsenen und Kindern gleich häufig auf [61]. Alle diese Beobachtungen wurden durch die Einschätzung der Cochrane-Gruppe bestätigt, dass sich die sublinguale Immuntherapie wegen ihrer guten Verträglichkeit zur selbständigen Behandlung durch den Patienten zu Hause eigne [5]. Neueren Untersuchungen zu Folge scheint das Auftreten von Nebenwirkungen bei der sublingualen Therapie im Bereich des 5- bis 500-fachen der subkutanen Dosis nicht dosisabhängig zu sein [62].

Die somit für die sublinguale Immuntherapie dokumentierte gute Verträglichkeit ist insbesondere im Vergleich zur subkutanen Immuntherapie von Relevanz, da letztere ein zwar geringes, aber doch vorhandenes Risiko für schwere Nebenwirkungen birgt. So wird in einer aktuellen Übersicht des Paul-Ehrlich-Instituts eine Inzidenz zwischen 0,02 und $0,007 \%$ pro verkaufter Packung nativer Allergenextrakte bzw. Allergoide für schwere, nicht-fatale, systemische Nebenwirkungen angegeben und in einem Zehnjahreszeitraum über das Auftreten von drei tödlich verlaufenden Zwischenfällen im Zusammenhang mit der subkutanen Applikation von Allergenextrakten berichtet [2].

\section{Literatur}

${ }^{1}$ Deutsche Gesellschaft für Allergologie und klinische Immunologie (DGAI), Ärzteverband Deutscher Allergologen (ÄDA), Deutsche Akademie für Allergologie und Umweltmedizin (DAAU). Weißbuch Allergie in Deutschland München: Medizin \& Wissen, 2004

${ }^{2}$ Lüderitz-Püchel U, Keller-Stanislawski B, Haustein D. Neubewertung des Risikos von Test- und Therapieallergenen. Bundesgesundheitsbl Gesundheitsforsch Gesundheitsschutz 2001; 44: 709-718

${ }^{3}$ Bousquet J, Lockey RF, Malling HJ. Allergen immunotherapy: therapeutic vaccines for allergic diseases (WHO position paper). Allergy 1998; 53 (No 44, suppl): 1 - 42

${ }^{4}$ Bousquet J and the ARIA Workshop Group. Allergic rhinitis and its impact on asthma. J Allergy Clin Immunol 2001; 108 (No 5, suppl): S147-S334

${ }^{5}$ Wilson DR, Torres Lima M, Durham S. Sublingual immunotherapy for allergic rhinitis. The Cochrane Library, 2003, Issue 2

${ }^{6}$ Kägi MK, Wüthrich B. Different methods of local allergen-specific immunotherapy. Allergy 2002; 57: 379-388

${ }^{7}$ Canonica GW, Passalacqua G. Noninjection routes for immunotherapy. J Allergy Clin Immunol 2003; 111: 437-448

${ }^{8}$ Malling HJ. Is sublingual immunotherapy clinically effective? Curr Opin Allergy Clin Immunol 2002; 2: $523-531$

${ }^{9}$ Kleine-Tebbe J, Bergmann KC, Bufe A et al. Aktueller Stellenwert der sublingualen Immuntherapie bei allergischen Krankheiten. Diskussionspapier. Allergo J 2004; 13: 430-434

${ }^{10}$ Durham S. Grass pollen tablet immunotherapy - an international multi-centre trial. Vortrag. XXIII Congress of the EAACI, 2004

${ }^{11}$ Jorde W, Linskens HF. Zur Persorption von Pollen und Sporen durch die intakte Darmschleimhaut. Acta allergol 1974; 29: 165-175 
12 Lens JW, Berg WB van den, Putte LBA van de et al. Flare-up of antigeninduced arthritis in mice after challenge with oral antigen. Clin Exp Immunol 1984; 58: $364-371$

13 Seifert J. Enterale Resorption großmolekularer Proteine bei Tieren und Menschen. Z Ernährungswissenschaft 1976; Suppl 18

14 Warshaw AL, Bellini CA, Walker WA. The intestinal mucosal barrier to intact antigenic protein. Difference between colon and small intestine. Am J Surg 1977; 133: 55 - 58

15 Udall JN, Walker WA. Antigentransport im Darm. Allergologie 1984; 7: 263-269

16 Jackson PG, Baker RWR, Lessof MH et al. Intestinal permeability in patients with eczema and food allergy. Lancet 1981; I: 1285 - 1286

17 Atherton DJ, Heddle RJ, Boulton P. Intestinal permeability in atopic eczema. Brit J Dermat 1984; 111 (suppl 26.7.1984): 34-35

18 Ukabam SO, Mann RJ, Cooper BT. Small intestinal permeability to sugars in patients with atopic eczema. Brit J Dermatol 1984; 110: 649- 652

${ }^{19}$ Holt PG, Sly PD. Emerging concepts of T-cell regulation in asthma and allergy. Allergy Clin Immunol Int - J World Allergy Org 2003; 15: $255-260$

${ }^{20}$ Secrist H, Chelen CJ, Wen Y et al. Allergen immunotherapy decreases IL-4 production in $\mathrm{CD}^{+} \mathrm{T}$ cells from allergic individuals. J Exp Med 1993; 178: $2123-2130$

21 Marcucci F, Sensi L, Di Cara G et al. Dose dependence of immunological response to sublingual immunotherapy. Erscheint in: Allergy,

${ }^{22}$ Noirey N, Rougier N, André C et al. Langerhans-like dendritic cells generated from cord blood progenitors internalise pollen allergens by macropinocytosis, and part of the molecules are processed and can activate autologous naïve T-lymphocytes. Clinical Allergy 2000; 13: $509-513$

${ }^{23}$ Hosken NA, Shibuya K, Heath AW et al. The effect of antigen dose on CD4+ T helper cell phenotype development in a T cell receptor-alpha beta-transgenic model. J Exp Med 1993; 182: 1579-1584

24 Tari MG, Mancino M, Monti G. Efficacy of sublingual immunotherapy in patients with rhinitis and asthma due to house-dust mites. A double-blind study. Allergol Immunopathol 1990; 8: 277-284

25 Troise C, Voltolini S, Canessa A et al. Sublingual immunotherapy in Parietaria pollen-induced rhinitis: a double blind study. J Investig Allergol Clin Immunol 1995; 5: 25 - 30

${ }^{26}$ Vourdas D, Syrigou E, Potamianou P et al. Double-blind, placebo-controlled evaluation of sublingual immunotherapy with standardized olive pollen extract in pediatric patients with allergic rhinoconjunctivitis and mild asthma due to olive pollen sensitization. Allergy 1998; 53: $662-672$

${ }^{27}$ Guez S, Vatrinet C, Fadel R et al. House-dust-mite sublingual-swallow immunotherapy (SLIT) in perennial rhinitis: a double-blind, placebocontrolled study. Allergy 2000; 55: $369-375$

28 Bousquet J, Scheinmann P, Guinnepain MT et al. Sublingual-swallow immunotherapy (SLIT) in patients with asthma due to house-dust mites: a double-blind, placebo-controlled study. Allergy 1999; 54: 249- 260

${ }^{29}$ Clavel R, Bousquet J, André C. Clinical efficacy of sublingual-swallow immunotherapy: a double-blind, placebo-controlled trial of a standardized five-grass-pollen extract in rhinitis. Allergy 1998; 53: 493-498

${ }^{30}$ La Rosa M, Ranno C, André C et al. Double-blind placebo-controlled evaluation of sublingual-swallow immunotherapy with standardized Parietaria judaica extract in children with allergy. J Allergy Clin Immunol 1999; 104: 425-432

${ }^{31}$ Falagiani P, Mistrello G. Pharmacokinetics of allergens after local administration. Allergy 1997; 52 (suppl 33): 17-21

${ }^{32}$ Mistrello G, Rapisarda G, Falagiani P. Detection of IgE-binding activity in serum after intranasal treatment of normal rabbits with P. judaica extract. Allergy 1991; 46: 52-58

33 Bagnasco M, Mariani G, Passalacqua G et al. Absorption and distribution kinetics of the major Parietaria judaica allergen (Par j1) administered by noninjectable routes in healthy human beings. J Allergy Clin Immunol 1997; 100: $122-129$

34 Voltolini S, Modena P, Minale P et al. Sublingual immunotherapy in tree pollen allergy. Double-blind, placebo-controlled study with a biologically standardised extract of three pollens (alder, birch and hazel) administered by a rush schedule. Allergol et Immunopathol 2001; 29: $103-110$

${ }^{35}$ Khinchi MS, Poulsen LK, Malling $\mathrm{HJ}$ et al. Clinical efficacy of sublingual and subcutaneous birch pollen allergen-specific immunotherapy: a randomized, placebo-controlled double-blind, double-dummy study. Allergy 2004; 59: 45-53

${ }^{36}$ Ariano R, Spandolini I, Panzani RC. Efficacy of sublingual specific immunotherapy in Cupressaceae allergy using an extract of Cupressus arizonica: a double blind study. Allergol Immunopathol (Madr) 2001; 29: $238-244$

37 Sabbah A, Hassoun S, Le Sellin J et al. A double-blind, placebo-controlled trial by the sublingual route of immunotherapy with a standardized grass pollen extract. Allergy 1994; 49: 309-313

38 Pradalier A, Basset D, Claudel A et al. Sublingual-swallow immunotherapy (SLIT) with a standardized five-grass-pollen extract (drops and sublingual tablets) versus placebo in seasonal rhinitis. Allergy 1999; 54: $819-828$

${ }^{39}$ Bufe A, Ziegler-Kirbach E, Stoeckmann E. Efficacy of sublingual swallow immunotherapy in children with severe grass pollen allergic symptoms: a double-blind placebo-controlled study. Allergy 2004; 59: $498-504$

${ }^{40}$ Rolinck-Werninghaus $\mathrm{C}$, Wolf $\mathrm{H}$, Liebke $\mathrm{C}$ et al. A prospective, randomized, double-blind, placebo-controlled multi-centre study on the efficacy and safety of sublingual immunotherapy (SLIT) in children with seasonal allergic rhinoconjunctivitis to grass pollen. Allergy 2004; 59: $1285-1293$

41 Smith H, White P, Annila I et al. Randomized controlled trial of highdose sublingual immunotherapy to treat seasonal allergic rhinitis. J Allergy Clin Immunol 2004; 114: 831 - 837

42 Torres Lima M, Wilson D, Pitkin L. Grass pollen sublingual immunotherapy for seasonal rhinoconjunctivis: a randomized controlled trial. Clin Exp Allergy 2002; 32: 507 - 514

43 Passalacqua G, Puccinelli P, Parmiani S et al. Clinical and immunologic effects of a rush sublingual immunotherapy to Parietaria species: a double-blind, placebo-controlled trial. J Allergy Clin Immunol 1999; 104: $964-968$

${ }^{44}$ André C, Perrin-Fayolle M, Grosclaude M et al. A double-blind placebo-controlled evaluation of sublingual immunotherapy with a standardized ragweed extract in patients with seasonal rhinitis. Int Arch Allergy Immunol 2003; 131: 111 - 118

${ }^{45}$ Hirsch Th, Sähn M, Leupold W. Double blind placebo controlled study of sublingual immunotherapy with house dust mite extract in children. Pediatr Allergy Immunol 1997; 8: 21 - 27

46 Bahceciler N, Isik U, Barlan I. Efficacy of sublingual immunotherapy in children with asthma and rhinitis: a double-blind, placebo-controlled study. Pediatric Pulmonology 2001; 32: 49-55

${ }^{47}$ Mortemousque B, Bertel F, De Casamayor J. House-dust-mite sublingual-swallow immunotherapy in perennial conjunctivitis: a doubleblind, placebo-controlled study. Clin Exp Allergy 2003; 33: 464-469

48 Tonnel AB, Scherpereel A, Douay B. Allergic rhinitis due to house dust mites: evaluation of the efficacy of specific sublingual immunotherapy. Allergy 2004; 59: 491 - 497

49 Merk HF, Sieber J. Safety of high-dose sublingual immunotherapy with ultra-rush titration - new follow-up data from a DBPC study in grasspollen allergic patients. XXIII Congress of the EAACI, 2004 (Abstract Book): 26

50 Vervloet D, Birnbaum J, Laurent P et al. Safety and clinical efficacy of rush sublingual Juniperus Ashei immunotherapy. XXII Congress of the EAACI, 2003 (Abstract Book): 228

51 Sieber J, Merk HF. Tolerability and effectiveness of seasonal SLIT with ultra-rush titration - an open prospective observational study. XXIII Congress of the EAACI, 2004 (Abstract Book): 140-141

52 Wilson DR, Walker S, Torres Lima M et al. Comparisons between injection and sublingual immunotherapy for hay fever: diary scores, skin responses, and serum antibodies. J Allergy Clin Immunol 2002; 109: S170

53 Mungan D, Misirligil Z, Gürbüz L. Comparison of the efficacy of subcutaneous and sublingual immunotherapy in mite-sensitive patients with rhinitis and asthma - a placebo controlled study. Ann Allergy Asthma Immunol 1999; 82: $485-490$

${ }^{54}$ Quirino T, Iemoli E, Siciliani E et al. Sublingual versus injective immunotherapy in grass pollen allergic patients: a double blind (double dummy) study. Clin Exp Allergy 1996; 26: 1253-1261

55 Bernardis P, Agnoletto M, Puccinelli P et al. Injective versus sublingual immunotherapy in Alternaria tenuis allergic patients. J Invest Allergol Clin Immunol 1996; 6: 55-62

56 Neumann D. Neues zur sublingualen Immuntherapie. Haut 2004; XV: $218-219$ 
${ }^{57}$ Di Rienzo V, Marcucci F, Puccinelli P et al. Long-lasting effect of sublingual immunotherapy in children with asthma due to house dust mite: a 10-year prospective study. Clin Exp Allergy 2003; 33: 206-210

${ }^{58}$ Novembre E, Galli E, Landi F et al. Coseasonal sublingual immunotherapy reduces the development of asthma in children with allergic rhinoconjunctivitis. J Allergy Clin Immunol 2004; 114: 851 - 857

${ }^{59}$ Marogna M, Spadolini I, Massolo A et al. Randomized controlled open study of sublingual immunotherapy for respiratory allergy in real-life: clinical efficacy and more. Allergy 2004; 59: 1205-1210
${ }^{60}$ Di Rienzo V, Pagani A, Parmiani S et al. Post-marketing surveillance study on the safety of sublingual immunotherapy in pediatric patients. Allergy 1999; 54: 1110-1113

${ }^{61}$ André C, Vatrinet C, Galvain S et al. Safety of sublingual-swallow immunotherapy in children and adults. Int Arch Allergy Immunol 2000; 121: $229-234$

62 Frati F, Incorvaia C, Gidaro G et al. Immunological changes in the oral mucosa during high dose sublingual immunotherapy. XXIII Congress of the EAACI, 2004 (Abstract Book): 93 - 94 